\title{
Leukocyte and Endothelial Activation in a Laboratory Model of Extracorporeal Membrane Oxygenation (ECMO)
}

\author{
JOHANNES GRAULICH, BARBARA WALZOG, MONIKA MARCINKOWSKI, KARL BAUER, \\ HANS KÖSSEL, GESA FUHRMANN, CHRISTOPH BÜHRER, PETER GAEHTGENS, \\ AND HANS T. VERSMOLD \\ Department of Neonatology, Charité School of Medicine, Campus Virchow, Humboldt Universität, Berlin \\ [J.G., C.B.]; and Departments of Pediatrics [M.M., K.B., H.K., H.T.V.] and Physiology [B.W., G.F., P.G.], \\ University Hospital Benjamin Franklin, Freie Universität Berlin, Berlin
}

\begin{abstract}
An inflammatory response and a capillary leak syndrome frequently develop during the treatment of neonatal respiratory failure by extracorporeal membrane oxygenation (ECMO). The present study was performed to investigate leukocyte activation and endothelial cell dysfunction that are associated with prolonged contact of blood components with synthetic surfaces. Laboratory ECMO was performed with fresh human blood at $37^{\circ} \mathrm{C}$ for $8 \mathrm{~h}(n=6)$. Leukocyte activation was measured by L-selectin (CD62L) and CD18 integrin surface expression and by neutrophil-derived elastase release. To monitor endothelial activation, endothelial cell ICAM-1 (CD54) expression was measured in cultured endothelial cells from human umbilical veins (HUVEC) after incubation with plasma from the ECMO experiments. CD18 integrin expression was found significantly upregulated on polymorphonuclear neutrophils and monocytes after 2-4 h of laboratory ECMO. L-selectin was reduced on both cell types during the total duration of the experiments. Soluble L-selectin (sCD62L) and total and differential leukocyte counts
\end{abstract}

\section{ABSTRACT}

remained unchanged during the experiment. Neutrophil-derived elastase content was maximal after $8 \mathrm{~h}$ of ECMO. Plasma from the ECMO experiments did not induce ICAM-1 expression of cultured HUVEC. We conclude that prolonged contact with synthetic surfaces during ECMO activates phagocytes, which may contribute to the inflammatory response seen in ECMOtreated patients. Activated phagocytes do not accumulate in the extracorporeal system nor release humoral factors inducing ICAM-1 expression on endothelial cells. (Pediatr Res 48: 679684, 2000)

\section{Abbreviations}

ECMO, extracorporeal membrane oxygenation

HUVEC, human umbilical vein endothelial cells

PMN, polymorphonuclear neutrophils

TNF- $\boldsymbol{\alpha}$, tumor necrosis factor $\alpha$

FMLP, N-formyl-Met-Leu-Phe
ECMO is the standard treatment for newborn infants with respiratory failure unresponsive to conventional pulmonary support (1). During ECMO treatment, an inflammatory reaction with neutropenia (2), activation of PMN (3), and a capillary leak syndrome with systemic and pulmonary edema $(4,5)$ have been described. However, it is not clear, to what extent this changes result from the patient's disease (6) or from the effects of extracorporeal circulation of the blood. We therefore performed laboratory ECMO without connecting the system to a patient, to study the isolated effects of prolonged extracorporeal circulation of blood on leukocyte number, expression of

Received August 18, 1999; accepted April 29, 2000.

Correspondence and reprint requests: Dr. Johannes Graulich, Klinik für Neonatologie, Humboldt Universität, Charité Campus Virchow Klinikum, Augustenburger Platz 1, D-13353 Berlin, Germany.

Supported by a grant of FSP Entzündliche Erkrankungen, Fachbereich Humanmedizin, Freie Universität Berlin, Germany. leukocyte adhesion molecules, and the release of mediators that might activate endothelial cells.

During hemodialysis or cardiopulmonary bypass (CPB), leukocytes are activated $(2,7,8)$ and humoral mediators are generated, which impairs endothelial cell integrity (9-11). The induction of an inflammatory response and the loss of endothelial integrity represent a hallmark of the capillary leakage commonly observed in CPB (7) and neonatal ECMO $(4,5)$, with systemic and pulmonary edema often prolonging the duration of ECMO. In spite of evidence for activation of PMN in neonatal ECMO (3), it is unclear to what extent this activation is due to the underlying disease or the leukocyte-synthetic surface interaction and whether this activation is linked to the clinically observed changes in endothelial cell barrier function.

In various animal models of pulmonary or cerebral inflammation, blockade of molecules mediating leukocyteendothelial cell adhesion has been shown to abrogate or alle- 
viate capillary plasma leakage $(8,12,13)$. These adhesion molecules include L-selectin (CD62L) and the CD11/CD18 integrins that are expressed by most leukocytes and are involved in leukocyte activation and recruitment to sites of inflammation. CD11/CD18 integrins are up-regulated upon activation (14), whereas, in contrast, L-selectin is rapidly shed (15), resulting in the generation of biologically active soluble L-selectin (sCD62L). SCD62L is present in the plasma of normal individuals (16) and is elevated during various pathologic conditions (17). In cord blood, diminished soluble and total cellular L-selectin is associated with its impaired ability of shedding surface L-selectin (18).

Intercellular adhesion molecule-1 (ICAM-1, CD54) serves as a receptor for $\mathrm{CD} 11 / \mathrm{CD} 18$ integrins and mediates leukocyte and endothelial cell adhesion and inflammatory vascular reactions (19). ICAM-1 expression is greatly increased by a variety of inflammatory stimuli (IL-1, tumor necrosis factor $\alpha$ (TNF$\alpha$ ), interferon $\gamma$, as well as bacterial endotoxins) (20) and serves as a marker for endothelial cell activation (14).

In the present study, we investigated whether blood circulated through an ECMO circuit at normothermic conditions for $8 \mathrm{~h}$ showed changes in leukocyte number and leukocyte activation (CD18 integrin surface up-regulation and L-selectin shedding, elastase release).

For testing whether endothelial activation occurs after contact with plasma of blood subjected to laboratory ECMO, we assayed ICAM-1 expression on cultured HUVEC. For positive control we incubated HUVEC with TNF- $\alpha$, which has been shown to be increased in an in vitro study of ECMO (21).

\section{MATERIALS AND METHODS}

Laboratory ECMO model. The same ECMO system used clinically for newborn ECMO patients suffering from respiratory failure was used in the present study. The circuit consisted of a silicone membrane oxygenator (0800 2, Avecor Cardiovascular Inc., Brooklyn Park, MN, USA), a heat exchanger (HEC 40, Jostra, Hirrlingen, Germany), 3/16 inch polyvinylchloride tubing (type Benjamin Franklin, Jostra), which is the standard tubing size for neonatal ECMO in our institution, and a nonocclusive roller pump (Jostra). This system, in contrast to circuits designed for newborns undergoing cardiac surgery, had no reservoir unit, therefore blood-air contact was minimal. The ECMO circuit had an absolute volume of $434 \pm 8 \mathrm{~mL}$ and was primed by the introduction of carbon dioxide, $0.9 \%$ saline solution, and $20 \%$ albumin. Subsequently, the system was filled with fresh (less than $24 \mathrm{~h}$ after venipuncture) human citrated phosphate dextrose (CPD) blood, from healthy adult donors. In agreement with a published report about adhesion molecule expression in cuprophane membrane hemodialysis (22), pilot experiments demonstrated that the addition of citrate to the samples did not change CD18 and L-selectin expression on PMN and monocytes. Heparin was added (300 IU/L). ECMO was performed at a constant temperature of $37^{\circ} \mathrm{C}$ and a constant flow rate of $300 \mathrm{~mL} / \mathrm{min}$. An appropriate mixture of air and carbon dioxide was supplied to the membrane oxygenator to keep blood gases in physiologic ranges. Electrolytes, $\mathrm{pH}$, and glucose were kept constant in the physiologic range during the experiments. Cells and plasma were separated by centrifugation. A total of six independent experiments were performed.

As control experiments, heparinized (300 IU/L) CPD blood was allowed to stand for $8 \mathrm{~h}$ at $37^{\circ} \mathrm{C}$ in the collecting bag of the venipuncture.

In laboratory ECMO and in control experiments EDTA blood samples were drawn at baseline and after $0.5,1,2,4$, and $8 \mathrm{~h}$ for analysis.

White blood cell counts. Total white blood cell counts were determined using an automated hematology analyzer (Coulter Electronics Inc., Hialeah, FL, USA) and absolute neutrophil, eosinophil, basophil, monocyte, and lymphocyte counts were calculated from results of Wright-Giemsa-stained blood smears.

Leukocyte adhesion molecules. Aliquots of whole blood (50 $\mu \mathrm{L}$ ) containing $2 \mathrm{mM}$ EDTA were incubated for $30 \mathrm{~min}$ at $4^{\circ} \mathrm{C}$ in the dark with indicated concentrations of FITC-labeled MAb MHM23 directed against human CD18 (Dako Diagnostica, Hamburg, Germany) and FITC-labeled MAb Leu-8 directed against human L-selectin (Becton Dickinson, Heidelberg, Germany) or FITC-labeled IgG2 isotype control (Dako Diagnostica). Lysis of erythrocytes was performed using FACS lysing solution (Becton Dickinson) according to the supplier's instructions. After final washing, cells were fixed with $1 \%$ paraformaldehyde. For positive control, an aliquot of blood (50 $\mu \mathrm{L}$ ) was stimulated with $100 \mathrm{nM}$ fMLP, a strong activator of PMN and monocytes at $37^{\circ} \mathrm{C}$. Samples were subjected to flow cytometry (FACScan, Becton Dickinson) as described (23). Twenty thousand cells were measured and gated offline for granulocytes, monocytes, and lymphocytes based on their different forward and side scatters, respectively. Median surface expression of L-selectin and CD18 was analyzed using the WinMDI version 2.4 software (http://facs.scripps.edu/). Surface expression of L-selectin, CD18, and ICAM-1 was calculated as mean of the median fluorescence intensity of six independent experiments. Soluble L-selectin (sCD62L) was determined in plasma using ELISA, as previously described (24).

Neutrophil-derived elastase. Neutrophil-derived elastase released from azurophilic granules of activated neutrophils into the plasma was measured as a marker of PMN activation by incubating thermoequilibrated plasma samples $(40 \mu \mathrm{L})$ with the same volume of elastase substrate solution containing 75 $\mu \mathrm{g}$ methoxysuccinyl-Ala-Ala-Pro-Val-para-nitroanilide for $1 \mathrm{~h}$ at $37^{\circ} \mathrm{C}(25,26)$. For positive and negative control, elastase content of plasma derived from fMLP-stimulated or -unstimulated whole blood was analyzed, respectively. OD was measured at $405 \mathrm{~nm}$ in a 96-well microtiter plate reader (MCC 340, Flow Laboratories, Meckenheim, Germany). All determinations were carried out in duplicate $(n=3)$.

ICAM-1 expression of endothelial cells. HUVEC were isolated as described in the literature (27) and cultured on fibronectin $(10 \mu \mathrm{g} / \mathrm{mL})$-coated culture dishes in MCDB 131 culture medium supplemented with $2 \%$ FCS, human recombinant basic fibroblast growth factor $(5 \mathrm{ng} / \mathrm{mL})$, human recombinant epidermal growth factor $(10 \mathrm{ng} / \mathrm{mL})$, heparin $(10$ $\mathrm{U} / \mathrm{mL})$, hydrocortisone $(1 \mu \mathrm{g} / \mathrm{mL})$, amphotericin B $(2.5 \mu \mathrm{g} /$ 
$\mathrm{mL})$, and gentamicin $(50 \mu \mathrm{g} / \mathrm{mL})$ in $5 \% \mathrm{CO}_{2}$ at $37^{\circ} \mathrm{C}$. ICAM-1 expression of cultured monolayers of confluent HUVEC of the first passage was determined after incubation with plasma that had passed through the ECMO circuit for $15 \mathrm{~min}, 30 \mathrm{~min}, 1 \mathrm{~h}$, $2 \mathrm{~h}, 4 \mathrm{~h}$, or $8 \mathrm{~h}$. Incubation with TNF- $\alpha(100 \mathrm{U} / \mathrm{mL})$ served as positive control. For flow cytometry, HUVEC were harvested with EDTA and incubated with the indicated amount of the FITC-labeled MAb 6.5B5 directed against human ICAM-1 (Dako Diagnostica). Cell surface expression of the antigen was analyzed as described above for leukocytes.

TNF- $\alpha$, heparin, hydrocortisone, MCDB 131 culture medium, and fMLP were obtained from Sigma Chemical Co. (Deisenhofen, Germany). Methoxysuccinyl-Ala-Ala-Pro-Valpara-nitroanilide was purchased from Bachem (Bubendorf, Switzerland). Fibronectin, FCS, human recombinant basic fibroblast growth factor, human recombinant epidermal growth factor, amphotericin B. and gentamicin were obtained from Biochrom (Berlin, Germany). FACS lysing solution was obtained from Becton Dickinson.

\section{Statistics}

Data are expressed as mean \pm SEM where applicable. Statistical significance was determined using Wilcoxon matched-pairs signed-ranks test (SPSS for Windows software, release 8.0.0, SPSS Inc., Chicago, IL, USA). $p<0.05$ was considered as significant.

\section{RESULTS}

Total and differential leukocyte counts during 8 h of laboratory ECMO (Table 1). Mean total white blood cell count was $4.0 \pm 0.5 \times 10^{3} / \mu \mathrm{L}$ at baseline. Absolute as well as relative numbers of leukocytes and their subgroups (PMN, eosinophils, monocytes, and lymphocytes) remained constant during the experiment. Basophils were less then $1 \%$ of leukocytes in all experiments (data not shown). Thus, there was no substantial loss of leukocytes by adhesion to the surface of the tubing or the oxygenator, or by mechanical cell destruction in the ECMO pump.

CD18 cell surface expression (Fig. 1). CD18 expression on PMN and monocytes increased significantly within $30 \mathrm{~min}$ after the onset of ECMO. On PMN, CD18 expression peaked after $4 \mathrm{~h}$ and declined subsequently. As positive control, an aliquot of blood was challenged with a maximal effective dose of fMLP (start of experiment: $155 \pm 20,4$ h ECMO: $256 \pm 36$, fMLP activation: $368 \pm 19$; fluorescence intensity in arbitrary units). On monocytes, maximum CD18 expression was observed already after $2 \mathrm{~h}$ of ECMO (start of experiment: $254 \pm$ 19, 2 h ECMO: $336 \pm 17$, fMLP activation: $348 \pm 22$ ). CD18

\section{CD18 expression}
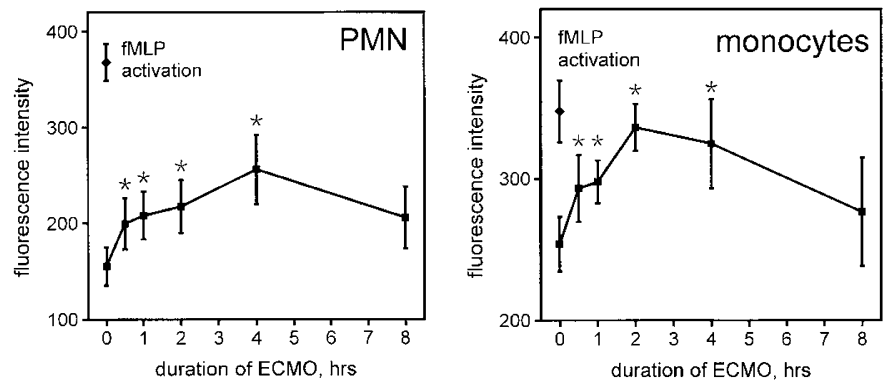

Figure 1. CD18 surface expression on PMN and monocytes was significantly up-regulated during laboratory ECMO. For positive control, blood was activated with $100 \mathrm{nM}$ fMLP for $5 \mathrm{~min}$ at $37^{\circ} \mathrm{C}$. Mean \pm SEM of six independent experiments. ${ }^{*} p<0.05$ vs baseline $(0 \mathrm{~h})$.

expression on lymphocytes showed no significant change after ECMO and fMLP activation (data not shown). Citrate phosphate dextrose (CPD) blood allowed to stand at $37^{\circ} \mathrm{C}$ for $8 \mathrm{~h}$ in the collecting bag of the venipuncture set containing heparin showed no activation of monocyte and PMN CD18 expression (Table 2).

L-selectin surface expression (Fig. 2). Within $4 \mathrm{~h}$ after the onset of ECMO, a significant down-regulation of L-selectin was detected on monocytes (start of experiment: $152 \pm 21,8 \mathrm{~h}$ ECMO: $41 \pm 8$, fMLP activation: $39 \pm 6$ ) and PMN (start of experiment: $166 \pm 20,8 \mathrm{~h}$ ECMO: $63 \pm 13$, fMLP activation: $37 \pm 10$ ). Down-regulation continued over $8 \mathrm{~h}$ of ECMO and almost reached the values induced by fMLP. The percentage of L-selectin-positive lymphocytes did not change significantly after fMLP stimulation or ECMO (data not shown). Stored heparinized CPD blood acting as control showed no downregulation of monocyte and PMN L-selectin expression (Table 2).

Soluble L-selectin (SCD62L) concentration in plasma. The sCD62L concentration in the plasma was $6.52 \pm 1.41 \mathrm{nmol} / \mathrm{L}$ (mean \pm SEM) at the start of the experiments and showed no significant change throughout the full time course of ECMO $(0.5$ h: $6.36 \pm 1.32,1$ h: $6.52 \pm 1.38,2$ h: $6.17 \pm 1.26,4$ h: $6.27 \pm 1.44,8 \mathrm{~h}: 6.43 \pm 1.29 \mathrm{nmol} / \mathrm{L}$; mean $\pm \mathrm{SEM})$.

Neutrophil-derived elastase concentration in plasma (Fig. 3). Elastase was measured in plasma derived from laboratory ECMO-treated blood. Neutrophil-derived elastase concentration was elevated significantly after $8 \mathrm{~h}$ of ECMO and was threefold enhanced as compared with untreated blood.

ICAM-1 expression on HUVEC. ICAM-1 expression on endothelial cells was not induced by incubation (up to $24 \mathrm{~h}$ ) with plasma derived from laboratory ECMO-treated blood (Fig. 4). This was true for plasma samples obtained after 0.5,

Table 1. Total and differential leukocyte counts during laboratory ECMO

\begin{tabular}{|c|c|c|c|c|c|c|}
\hline & Baseline & $0.5 \mathrm{~h}$ & $1 \mathrm{~h}$ & $2 \mathrm{~h}$ & $4 \mathrm{~h}$ & $8 \mathrm{~h}$ \\
\hline Total white blood cells $\left(10^{3} / \mu \mathrm{L}\right)$ & $4.0 \pm 0.5$ & $3.5 \pm 0.5$ & $3.6 \pm 0.6$ & $3.7 \pm 0.5$ & $3.6 \pm 0.5$ & $4.0 \pm 0.8$ \\
\hline $\operatorname{PMN}\left(10^{3} / \mu \mathrm{L}\right)$ & $1.5 \pm 0.2$ & $1.5 \pm 0.3$ & $1.3 \pm 0.3$ & $1.4 \pm 0.4$ & $1.4 \pm 0.3$ & $1.5 \pm 0.3$ \\
\hline Eosinophils $\left(10^{3} / \mu \mathrm{L}\right)$ & $0.2 \pm 0.07$ & $0.11 \pm 0.02$ & $0.11 \pm 0.03$ & $0.28 \pm 0.14$ & $0.24 \pm 0.11$ & $0.16 \pm 0.06$ \\
\hline Monocytes $\left(10^{3} / \mu \mathrm{L}\right)$ & $0.19 \pm 0.06$ & $0.18 \pm 0.07$ & $0.14 \pm 0.06$ & $0.14 \pm 0.06$ & $0.2 \pm 0.08$ & $0.15 \pm 0.06$ \\
\hline
\end{tabular}

Mean $\pm \mathrm{SEM}, n=6$. 
Table 2. CD18 and L-selectin expression in control experiments

\begin{tabular}{|c|c|c|c|c|c|c|}
\hline & Baseline & $0.5 \mathrm{~h}$ & $1 \mathrm{~h}$ & $2 \mathrm{~h}$ & $4 \mathrm{~h}$ & $8 \mathrm{~h}$ \\
\hline CD18 on PMN & $140 \pm 18$ & $143 \pm 9$ & $147 \pm 6$ & $104 \pm 3$ & $106 \pm 8$ & $100 \pm 12$ \\
\hline CD18 on monocytes & $237 \pm 18$ & $234 \pm 11$ & $231 \pm 17$ & $258 \pm 20$ & $224 \pm 21$ & $200 \pm 30$ \\
\hline L-selectin on PMN & $152 \pm 7$ & $137 \pm 6$ & $146 \pm 5$ & $137 \pm 6$ & $132 \pm 7$ & $150 \pm 11$ \\
\hline
\end{tabular}

Mean fluorescence intensity in arbitrary units \pm SEM, $n=6$.
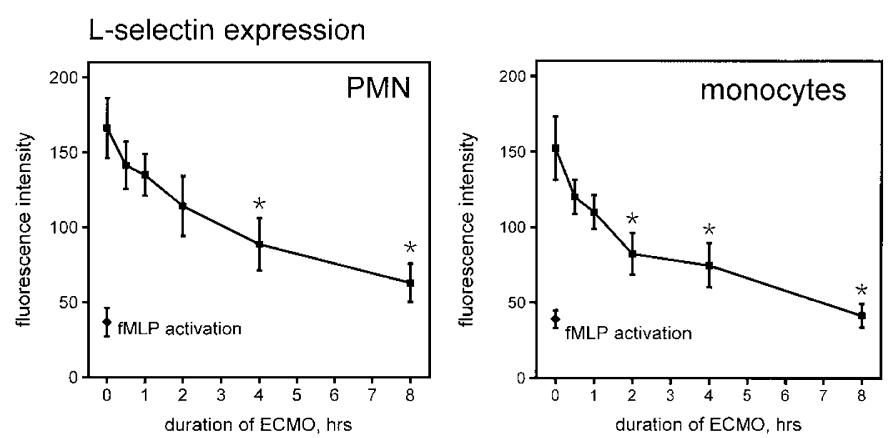

Figure 2. L-selectin surface expression on PMN and monocytes was significantly down-regulated during laboratory ECMO. For positive control, blood was activated with $100 \mathrm{nM}$ fMLP for $5 \mathrm{~min}$ at $37^{\circ} \mathrm{C}$. Mean \pm SEM of six independent experiments. ${ }^{*} p<0.05 v s$ baseline $(0 \mathrm{~h})$.

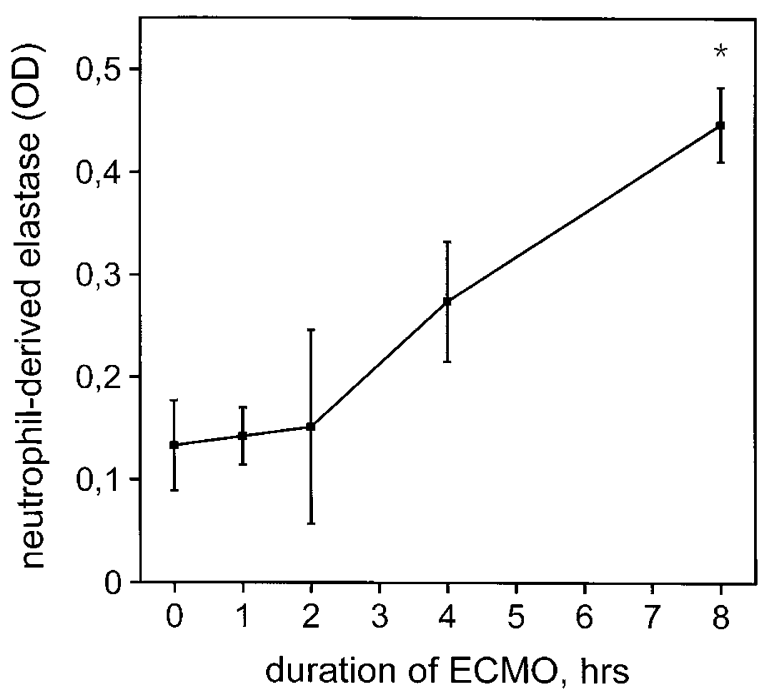

Figure 3. Neutrophil-derived elastase concentration measured as OD increased with the duration of ECMO and is significantly elevated after $8 \mathrm{~h}$ (mean $\pm \mathrm{SEM}) .{ }^{*} p<0.05 v s$ baseline $(0 \mathrm{~h})$.

1, 2, 4, or $8 \mathrm{~h}$ of laboratory ECMO. For positive control, expression of ICAM-1 was induced by TNF- $\alpha$. This effect was time- and dose-dependent with maximal expression at $8 \mathrm{~h}$ after stimulation in the presence of $100 \mathrm{U} / \mathrm{mL}$ TNF- $\alpha$. Moreover, ECMO-derived plasma samples failed to synergize with a submaximal effective concentration of TNF- $\alpha(10 \mathrm{U} / \mathrm{mL})$ in inducing ICAM-1 expression (data not shown).

\section{DISCUSSION}

We used a laboratory model of ECMO appropriate for newborns to study the potential mechanisms for the leukocyte depletion, leukocyte activation, and endothelial dysfunction observed in neonates placed on ECMO. ECMO is used for the treatment of neonates with respiratory disease unresponsive to

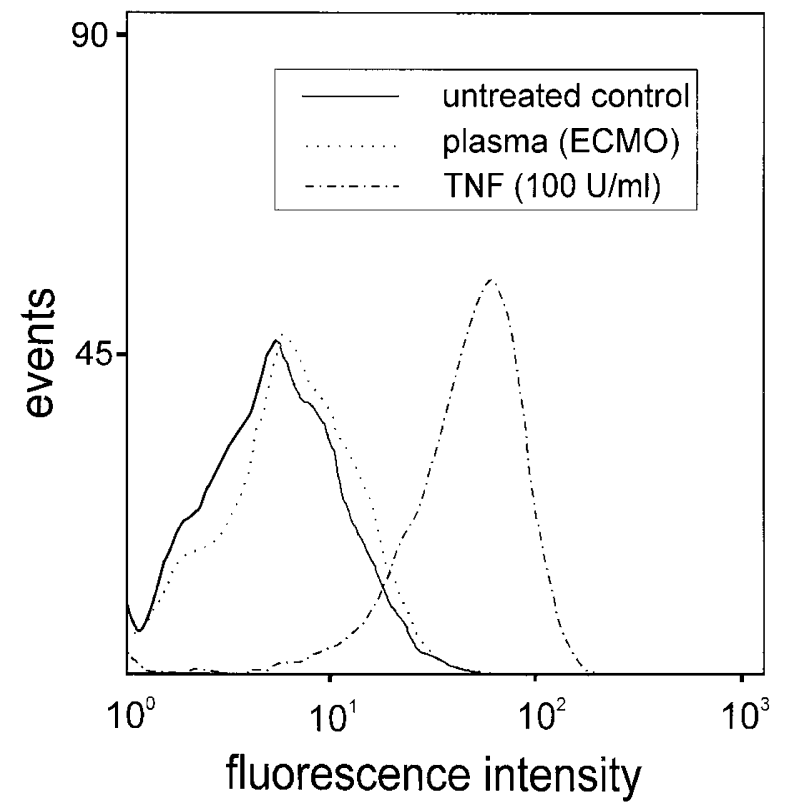

Figure 4. Typical fluorescence histogram of ICAM-1 expression on HUVEC after contact with plasma from blood circulated for $8 \mathrm{~h}$ through the ECMO system. Positive control experiment with $100 \mathrm{U} / \mathrm{mL}$ TNF- $\alpha$. The effect of TNF- $\alpha$ was time- and dose-dependent.

conventional treatment. In contrast to $\mathrm{CPB}$, lasting for few hours, ECMO lasts for several days and is performed under normothermic conditions.

During ECMO, capillary leaks with systemic and pulmonary edema are common $(4,5)$. A large number of factors have been implicated in the development of capillary leak syndrome in CPB (28). During ECMO, treatment effects of the patient's underlying disease and the hypoxia before initiation of ECMO and effects of the extracorporeal circulation of blood cannot be analyzed separately. Therefore we used a laboratory ECMO model to study the isolated effects of the extracorporeal circulation of blood.

Previous studies of leukocyte activation during laboratory CPB focused on short-term changes of PMN. Finn et al. demonstrated PMN L-selectin shedding during $2 \mathrm{~h}$ of laboratory CPB (29). Observing for longer time periods, we demonstrate continued L-selectin shedding over $8 \mathrm{~h}$ on PMN in laboratory ECMO. CD18 up-regulation on PMN was maximal after $4 \mathrm{~h}$. After transfusion of filtration-leukapheresis PMN to granulocytopenic patients with leukemia, functional skinchamber studies showed PMN adhesion at sites of inflammation for at least $6 \mathrm{~h}$ after transfusion (30).

In addition to PMN, we studied monocyte and lymphocyte activation. Lymphocyte L-selectin and CD18 expression were not changed significantly in our laboratory ECMO model. But, changes in adhesion molecule expression of monocytes were 
even more pronounced than in neutrophils. These changes of cell surface expression of monocytes by laboratory ECMO were comparable with fMLP-induced values for both L-selectin and CD18.

We did not observe an increase of soluble L-selectin (sCD62L) concentrations despite the significant L-selectin shedding from PMN and monocytes. This may be explained by the relation of L-selectin molecules per cell (31), the limited leukocyte number in our extracorporeal system, and the high concentration of soluble L-selectin in human plasma. In contrast to the clinical situation, where the pool of circulating leukocytes can be affected by demargination from endothelial surfaces or by immigration from bone marrow, no new leukocytes enter the circulating pool in laboratory ECMO. Therefore, changes in sCD62L in our model are not affected by leukocyte turnover or by endothelial sequestration in microvascular beds, which has been postulated to account for the low sCD62L plasma concentrations observed in early stages of acute respiratory distress syndrome (17).

Although phagocytes were profoundly activated in our model, we observed no substantial white blood cell loss in the ECMO circulation during the experiments. This is different from observations of neutropenia and monocytopenia in patients undergoing ECMO therapy $(11,32)$. Zach et al. described substantial leukopenia during neonatal ECMO compared with neonates without ECMO support (33) and speculated that this effect could be caused by adherence of leukocytes within the ECMO circuit or tubing. In our model of laboratory ECMO, no evidence was obtained to support this hypothesis. The explanation for the discrepancy between the observation of no leukopenia during laboratory ECMO and leukopenia during ECMO therapy of a patient could be that activated leukocytes do not adhere on the synthetical surface of the oxygenator or tubing but adhere to the endothelium of the patient's blood vessels or that activated leukocytes are recruited to the patient's tissues. This could contribute to the mechanisms of edema formation in neonatal ECMO patients.

A variety of proinflammatory soluble factors can impair endothelial integrity and induce a capillary leak syndrome (34). Such soluble factors are released during in vivo ECMO and in vitro models of ECMO (21). Thus, it was surprising that incubation of HUVEC with plasma did not lead to ICAM-1 up-regulation of endothelial cells in our experiments. Although this may be due to instability of a putative factor causing endothelial changes, we speculate that direct contact between activated leukocytes and endothelial cells is necessary to induce such changes of endothelial function. However, we did not measure levels of proinflammatory cytokines, e.g. IL-1, IL6 , IL-8, or TNF- $\alpha$. Further investigations will be necessary to clarify the exact extent and mechanism by which ECMO treatment causes the loss of endothelial integrity that undoubtedly exists in neonatal ECMO patients suffering from capillary leak syndrome.

In our study we found strong evidence that the extracorporeal circulation of blood does not induce neutropenia, but a long-lasting and profound phagocyte activation. Considering that we did not find evidence for the release of soluble factors in the plasma by ECMO with impact on endothelial ICAM-1 adhesion molecule expression, future studies should focus on direct leukocyte-endothelial interaction as a possible mechanism by which ECMO treatment causes the loss of endothelial integrity.

Acknowledgments. The authors thank Dietger Stiebenz for his help in measuring sCD62L. The excellent technical assistance of Mrs. G. Beyer, Mrs. W. Jekabsons, and Mrs. E. Strauss is gratefully acknowledged.

\section{REFERENCES}

1. UK Collaborative ECMO Trial Group 1996 UK collaborative randomised trial of neonatal extracorporeal membrane oxygenation. Lancet 348:75-82

2. Cameron D 1996 Initiation of white cell activation during cardiopulmonary bypass: cytokines and receptors. J Cardiovasc Pharmacol 27:S1-S5

3. Fortenberry JD, Bhardwaj V, Niemer P, Cornish JD, Wright JA, Bland L 1996 Neutrophil and cytokine activation with neonatal extracorporeal membrane oxygenation. J Pediatr 128:670-678

4. Anderson III HL, Coran AG, Drongowski RA, Ha HJ, Bartlett RH 1992 Extracellular fluid and total body water changes in neonates undergoing extracorporeal membrane oxygenation. J Pediatr Surg 27:1003-1008

5. Kelly Jr RE, Phillips JD, Foglia RP, Bjerke HS, Barcliff LT, Petrus L, Hall TR 1991 Pulmonary edema and fluid mobilization as determinants of the duration of ECMO support. J Pediatr Surg 26:1016-1022

6. Peek GJ, Firmin RK 1999 The inflammatory and coagulative response to prolonged extracorporeal membrane oxygenation. ASAIO J 45:250-263

7. Finn A, Naik S, Klein N, Levinsky RJ, Strobel S, Elliott M 1993 Interleukin-8 release and neutrophil degranulation after pediatric cardiopulmonary bypass. J Thorac Cardiovasc Surg 105:234-241

8. Granert C, Raud J, Xie X, Lindquist L, Lindbom L 1994 Inhibition of leukocyte rolling with polysaccharide fucoidin prevents pleocytosis in experimental meningitis in the rabbit. J Clin Invest 93:929-936

9. Downing SW, Edmunds LH 1992 Release of vasoactive substances during cardiopulmonary bypass. Ann Thorac Surg 54:1236-1243

10. Seghaye MC, Grabitz RG, Duchateau J, Busse S, Dabritz S, Koch D, Alzen G, Hornchen H, Messmer BJ, Von Bernuth G 1996 Inflammatory reaction and capillary leak syndrome related to cardiopulmonary bypass in neonates undergoing cardiac operations. J Thorac Cardiovasc Surg 112:687-697

11. Sinclair DG, Haslam PL, Quinlan GJ, Pepper JR, Evans TW 1995 The effect of cardiopulmonary bypass on intestinal and pulmonary endothelial permeability. Chest 108:718-724

12. Mulligan MS, Miyasaka M, Tamatani T, Jones ML, Ward PA 1994 Requirement for L-selectin in neutrophil-mediated lung injury in rats. J Immunol 152:832-840

13. Mulligan MS, Vaporciyan AA, Warner RL, Jones ML, Foreman KE, Miyasaka M, Todd III RF, Ward PA 1995 Compartmentalized roles for leukocytic adhesion molecules in lung inflammatory injury. J Immunol 154:1350-1363

14. Dustin ML, Springer TA 1988 Lymphocyte function associate antigen (LFA-1) interaction with intercellular adhesion molecule-1 (ICAM-1) is one of at least three mechanisms of lymphocyte adhesion to culture endothelial cell. J Cell Biol 107:321331

15. Kishimoto TK, Jutila MA, Berg EL, Butcher EC 1989 Neutrophil MAC-1 and MEL-14 adhesion proteins inversely regulated by chemotactic factors. Science 245:1238-1241

16. Schleiffenbaum B, Spertini O, Tedder TF 1992 Soluble L-selectin is present in human plasma at high levels and retains functional activity. J Cell Biol 119:229-238

17. Donnelly SC, Haslett C, Dransfield I, Robertson CE, Carter DC, Ross JA, Grant IS, Tedder TF 1994 Role of selectins in the development of adult respiratory distress syndrome. Lancet 344:215-219

18. Koenig JM, Simon J, Anderson DC, Smith E, Smith CW 1996 Diminished soluble and total cellular L-selectin in cord blood is associated with its impaired shedding from activated neutrophils. Pediatr Res 39:616-621

19. Argenbright LW, Barton RW 1992 Interactions of leukocyte integrins with intercellular adhesion molecule 1 in the production of inflammatory vascular injury in vivo. J Clin Invest 89:259-272

20. Pohlman TH, Stanness KA, Beatty PG, Ochs HD, Harlan JM 1986 An endothelial cell surface factor induced in vitro by lipopolysaccharide, interleukin-1, and tumor necrosis factor increases neutrophil adherence by a Cdw18-dependent mechanism. J Immunol 136:4548-4553

21. Adrian K, Mellgren K, Skogby M, Friberg LG, Mellgren G, Wadenvik H 1998 Cytokine release during long-term extracorporeal circulation in an experimental model. Artif Organs 22:859-863

22. Dhondt A, Vanholder R, Waterloos MA, Glorieux G, De Smet R, Lameire N 1998 Citrate anticoagulation does not correct cuprophane bioincompatibility as evaluated 
by the expression of leukocyte surface molecules. Nephrol Dial Transplant 13:17521758

23. Bührer C, Graulich J, Stibenz D, Dudenhausen JW, Obladen M 1994 L-selectin is down-regulated in umbilical cord blood granulocytes and monocytes of newborn infants with acute bacterial infection. Pediatr Res 36:799-804

24. Bührer C, Stibenz D, Graulich J, Gernhold U, Butcher EC, Dudenhausen JW, Obladen M 1995 Soluble L-selectin (sCD62L) umbilical cord plasma levels increase with gestational age. Pediatr Res 38:336-341

25. Borregard N, Cowland JB 1997 Granules of the human neutrophilic polymorphonuclear leukocyte. Blood 89:3503-3521

26. Tapper H 1996 The secretion of preformed granules by macrophages and neutrophils J Leukocyte Biol 59:613-622

27. Jaffé EA, Nachmann RL, Becker CG, Minick CR 1973 Culture of human umbilical endothelial cells derived from umbilical veins. Identification by morphologic and immunologic criteria, J Clin Invest 52:2745-2756

28. Ashraf SS, Tian Y, Zacharrias S, Cowan D, Martin P, Watterson K 1997 Effects of cardiopulmonary bypass on neonatal and paediatric inflammatory profiles. Eur J Cardiothorac Surg 12:862-868
29. Finn A, Rebuck N, Moat N 1992 Neutrophil activation during cardiopulmonary bypass (letter). J Thorac Cardiovasc Surg 102:1746-1748

30. Alavi JB, Root RK, Djerassi I, Evans AE, Gluckman SJ, MacGregor RR, Guerry D, Schreiber AD, Shaw JM, Koch P, Cooper RA 1977 A randomized clinical trial of granulocyte transfusions for infection in acute leukemia. N Engl J Med 296:706-711

31. Rebuck N, Finn A 1994 Polymorphonuclear granulocyte expression of CD11a/CD18, CD11b/CD18 and L-selectin in normal individuals. FEMS Immunol Med Microbio 8:189-195

32. Hocker JR, Wellhausen SR, Ward RA, Simpson PM, Cook LN 1991 Effect of extracorporeal membrane oxygenation on leukocyte function in neonates. Artif Organs 15:23-28

33. Zach TL, Steinhorn RH, Georgieff MK, Mills MM, Green TP 1990 Leukopenia associated with extracorporeal membrane oxygenation in newborn infants. J Pediatr 116:440-444

34. Burke-Gaffney A, Keenan AK 1993 Modulation of human endothelial cell permeability by combinations of the cytokines interleukin- 1 alpha/beta, tumor necrosis factor-alpha and interferon-gamma. Immunopharmacology 25:1-9 